\title{
A Blind Handover Technique for the Intelligent Transportation Network
}

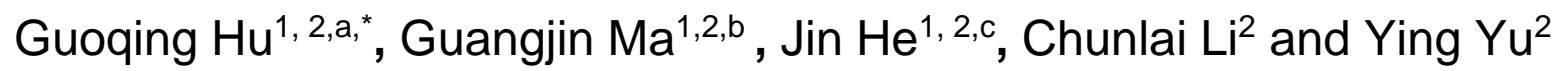 \\ ${ }^{1}$ School of Electronics Engineering and Computer Science, Peking University, Beijing, China \\ 2 Peking University Shenzhen SoC Key Laboratory, Peking University Shenzhen Institution, \\ Shenzhen, China
}

ahuking@pku.edu.cn, bguangjin.ma@pku.edu.cn, cfrankhe@pku.edu.cn

${ }^{*}$ Corresponding author: huking@pku.edu.cn

\begin{abstract}
Keywords: Blind Handover, Mobile Communications, Intelligent Transportation Network.
Abstract. In order to solve the problem of frequent handoff in intelligent transportation networks, a novel blind handover technique is proposed. The technology is based on the vehicle moving route reported by the vehicle navigation system, generating the target cell database and handover trigger location database. When the vehicle navigation GPS and the mobile network perceive the vehicle arriving at the handover trigger point, the system automatically extracts the corresponding target cell, and then completes the handover. Unlike traditional handover methods, the proposed technique does not require handover measurements, greatly reduces the total handover latency, and improves the handover success rate and service quality.
\end{abstract}

\section{一种智能交通网中的盲切换技术 \\ 胡国庆 ${ }^{1,2}, a^{*}{ }^{*}$,马光金 ${ }^{1,2, b}$,何进 ${ }^{1,2, c}$,李春来 ${ }^{2}$, 余迎 ${ }^{2}$ \\ 1 北京大学信息科学技术学院, 北京, 中国 \\ 2北京大学深圳研究院系统芯片设计重点实验室, 深圳, 中国}

ahuking@pku.edu.cn, bguangjin.ma@pku.edu.cn, cfrankhe@pku.edu.cn

*通讯作者: huking@pku.edu.cn

关键词:盲切换; 移动通信; 智能交通网。

中文摘要. 为解决智能交通网中存在的移动台频繁切换问题, 本文提出了一种新型的盲切换 技术。该技术基于车载导航系统上报的车辆移动路线, 生成车辆待切换目标小区数据库和切 换触发地点数据库。当车载导航GPS和移动网感知到车辆到达切换触发地点时, 系统自动从 切换目标小区数据库中提取对应的目标小区, 进而完成切换。与传统的切换方法不同, 该技 术无需进行切换测量, 大幅降低了车载乘客的总的切换时延, 提升了车载移动用户的切换成 功率和服务质量。

\section{1. 引言}

根据中国国家统计局的统计资料显示：2016年年末，全国机动车保有量达2.9亿辆，其中 汽车1.94亿辆。乘坐车辆出行, 已经成了人们生活中不可或缺的生活方式。随着智能手机终 端的普及, 人们在乘车出行时, 已经习惯于使用手机智能终端接入互联网的服务。为了给数 量庞大的手机智能终端用户 (截止到2017年6月, 中国移动用户: 8.67 亿, 中国联通用户: 2.69 亿, 中国电信： 2.30 亿）提供互联网接入服务, 移动运营商必须提供给用户更大的带宽。为 此, 必须尽量缩小蜂窝小区的半径, 以提供更大的带宽。但对快速移动中的车载乘客而言, 较小的小区半径，就意味着更加频繁的切换 ${ }^{[1]-[3] 。}$ 
如图1所示, 传统的切换流程分为切换测量(Handover Measurement)、切换判决(Handover Decision)、切换执行(Handover Execution) 三步[4]-[5]。所谓切换测量是指用户端设备UE(User Equipment)对附近的若干个相邻小区的信号质量进行测量, 基站根据测量报告选择信号质量 最好的小区作为切换目标小区。这种测量方式需要大量的测量时间, 以WCDMA和LTE为例, 整个切换测量时间需要 $200 \mathrm{~ms}$ 以上 ${ }^{[6]}$ 。在切换判决前, 为了防止乒乓效应, 还必须有一段切

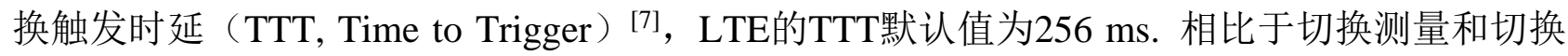
判决所花费的时间, LTE切换执行的时间仅需 $100 \mathrm{~ms}$.

综上分析，切换测量和切换触发时延相对于切换执行时间占了太大的比重，为了进一步 缩短总的切换时延，必须尽量切换测量和触发时延所占用的时间。本文基于车载导航系统上 报的车辆移动路线以及车辆的运行特征, 设计了一种智能交通网中的盲切换技术。利用该方 案, 可以无需切换测量和切换判决时延, 进而大幅降低车载乘客的切换时延, 提升移动用户 的切换成功率和服务质量。

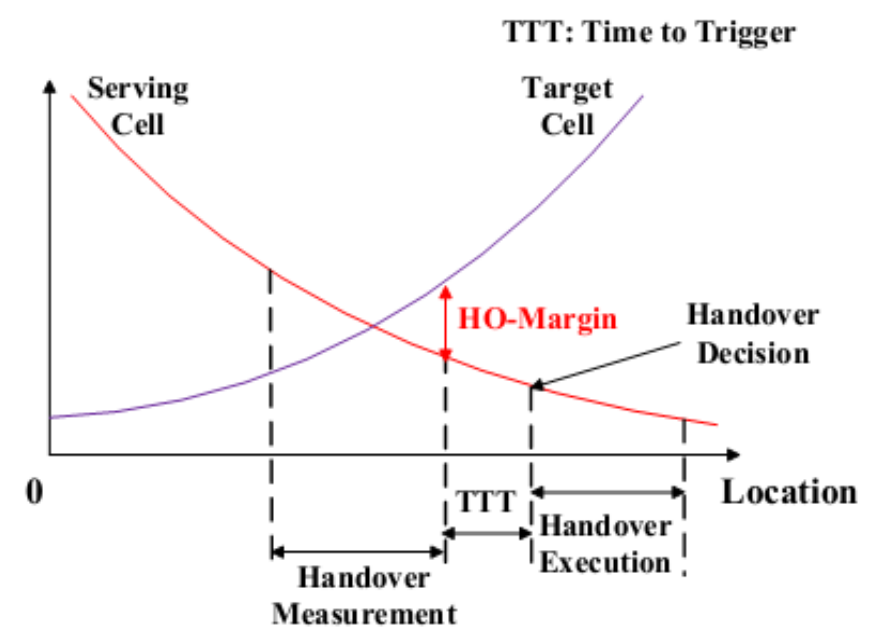

图1 传统切换流程图

\section{2. 智能交通网盲切换方案}

本方案的主要特点是将传统移动台辅助切换转换为系统独立完成切换, 可以无需切换测 量和切换判决时延, 所以称之为盲切换。

本发明的技术方案如下，图2为本方案的流程图:

\section{(1) 车载导航系统上报车辆移动路线}

车载导航系统根据目的地选择合适的移动路线, 并将该移动路线上报给临近的基站, 由 基站将移动路线上报给基站控制中心。

\section{(2) 生成车辆待切换目标小区数据库及切换触发地点数据库}

车辆待切换目标小区数据库建立过程如下: 基站控制中心根据车辆上报的移动路线, 判 断车辆即将经过的目标小区, 并将这些小区按照先后经过的顺序建立待切换目标小区数据库。

切换触发地点数据库建立过程如下: 如图3所示, 沿着车辆移动路线, 根据测量数据或切 换历史记录得出所有相邻小区之间信号质量相等的切换触发地点 $\mathrm{M}$, 生成切换触发地点数据 库。根据车辆的移动方向, 建立切换触发地点 $\mathrm{M}$ 与目标小区间的对应关系。

\section{(3) 利用车载导航GPS系统和移动网联合确定车辆及移动台的位置}

利用车载导航GPS系统和移动网联合确定车辆及移动台的位置, 并将该位置信息上报给 基站控制中心。

(4) 根据切换触发地点数据库, 判定车辆是否到达切换触发地点 
基站控制中心根据上报的车辆位置信息, 判断车辆是否到达切换触发地点。一旦车辆到 达触发地点 $\mathrm{M}$, 系统立即触发切换, 开始选择切换目标小区; 若未到达小区边缘, 返回步骤 2 , 继续探测车辆位置, 等待车辆到达触发地点。

\section{(5) 根据车辆待切换目标小区数据库, 选择切换目标小区}

车辆首先从待切换目标小区数据库提取它的候选小区列表，一旦车辆到达相邻小区的触 发切换地点 $\mathrm{M}$ ，则从候选小区列表中提取该触发地点所对应的目标小区。

\section{(6) 系统将同一车辆内的所有移动用户切换到目标小区}

由于同一车辆内的移动用户的运动规律是一致的, 所以他们的目标小区也是相同的。系 统将同一车辆内的移动台同时切换到目标小区。

切换完成后, 返回步骤 (2), 重新确定车辆位置, 等待车辆到达下一个切换触发地点。 以此递推，车辆被不断地切换到新的目标小区。

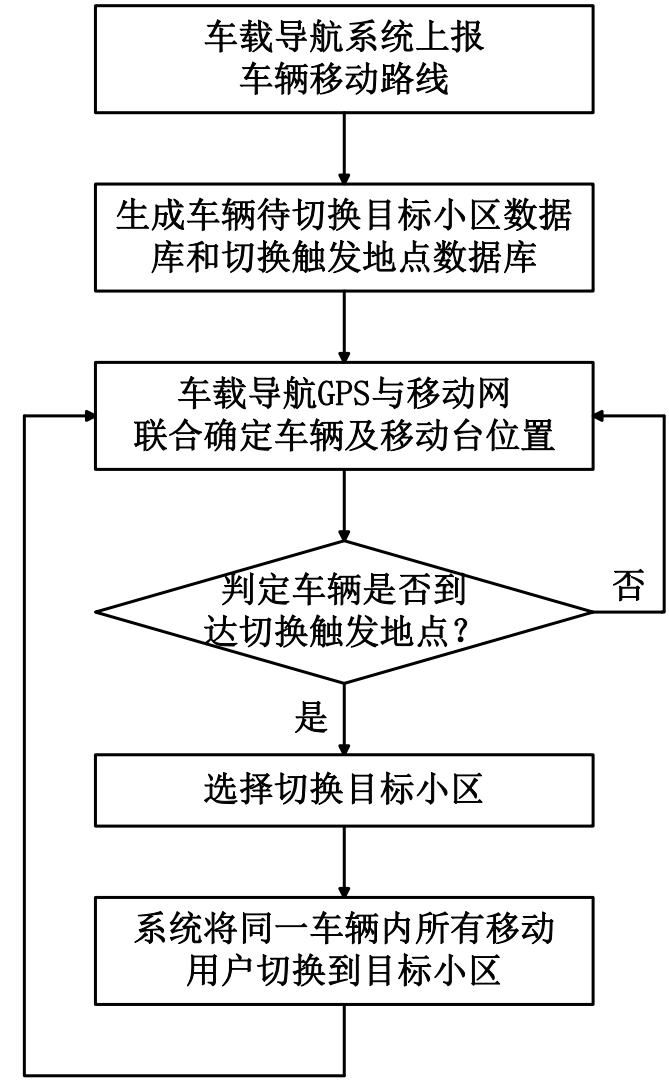

图2 智能交通网盲切换流程图

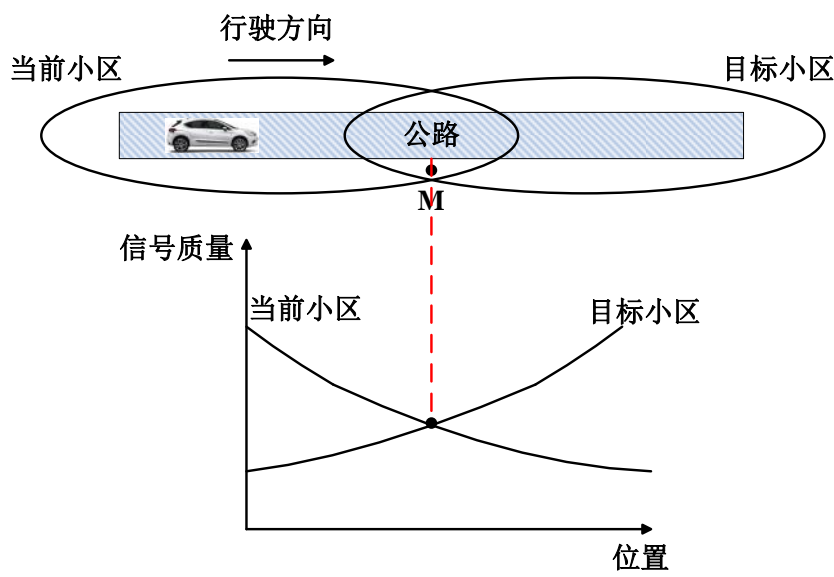

图3 小区边缘切换触发地点说明图 


\section{3. 结束语}

与现有的传统切换方法不同, 本方案无需切换测量, 而是由系统独立完成切换。如此一 来，就直接在总的切换时延中砍掉了信号质量测量时间及切换触发时延，大幅降低了总的切 换时延, 极大地提高了切换成功率和无线接入的服务质量, 对于通信运营商及智能交通网有 着重大的产业意义。

\section{致谢}

本文由深圳市基础研究项目（ JCYJ20170307164201104、JCYJ20160329161334453、 JCYJ20170412153845293、JCYJ20170307164247428、JSGG20170414140411874），国家自然科 学基金项目（61574005）, 深港产学研基地产学研项目（场效应太赫兹器件的关键技术研究） 资助。

\section{References}

[1] Guoqing Hu, Anpeng Huang, Tammy Chang, Xiang Cheng, Hequan Wu, LinzhenXie, Anshi $\mathrm{Xu}$, Zhangyuan Chen, "A Sensor-Based Seamless Handover Solution for Express Train Access Networks (ETANs),” IEEE Communications Letters, vol.16, no.4, pp. 470-472, April 2012.

[2] Guoqing Hu, Anpeng Huang, Ruisi He, Bo Ai, Zhangyuan Chen, "Theory analysis of the handover challenge in Express Train Access Networks (ETAN)", China Communications, vol. 11, no.7, pp. 92-98, July 2014.

[3] Guoqing $\mathrm{Hu}$, Guangjin Ma, Chuanlai Li, Yongchi Xu, Jin He, Ying Yu, Yandong He, “An Empirical Analysis of the Radio Propagation Characteristics in High-Speed Railway Environment", 2017 International Conference on Electrical, Mechanical and Computer Engineering (ICEMCE 2017), IET, Chengdu, China, pp.1-4, May 25-28, 2017.

[4] Cheng-Xiang Wang, Ammar Ghazal, Bo Ai, et al., "Channel Measurements and Models for High-Speed Train Communication Systems: A Survey," IEEE Communications Surveys \& Tutorials, vol. 18, no. 2, pp. 974-987, 2016.

[5] Guoqing Hu, Jin He, Guangjin Ma, Chunlai Li , Ying Yu, "Summary of the Study on High Speed Railway Access Network", The 2017 2nd International Conference on Electrical Technology and Mechanical Engineering (ICETME 2017), Guangzhou, China, pp.1-4, July 15-16, 2017.

[6] A. Ghazal, Y. Yuan, C.-X. Wang*, Y. Zhang, Q. Yao, Y. Yuan, H. Zhou, and W. Duan, "A non-stationary IMT-A MIMO channel model for high-mobility wireless communication systems,” IEEE Trans. Wireless Commun., vol. 16, no. 4, pp. 2057-2068, Apr. 2017.

[7] J. Huang, C.-X. Wang*, R. Feng, J. Sun, W. Zhang, and Y. Yang, "Multi-frequency MmWave massive MIMO channel measurements and characterization for $5 \mathrm{G}$ wireless communication systems,” IEEE J. Sel. Areas Commun., vol. 35, no. 7, pp. 1591-1605, July 2017. 